\title{
Economic and environmental optimal operation of isolated multi-element complementary microgrid based on PSO with adaptive mutation
}

\author{
Zhu Weiping ${ }^{1, ~ a ~}$, Yan Shuchang ${ }^{2, \text { b }}$, Wang Yulong ${ }^{2, c}$, Li Peng ${ }^{2, ~ d, H a n ~ P e n g f e i ~}{ }^{2, \text { e }}$ \\ 1 Jiangsu Electric Power Company Research Institute, Nanjing, 211013, China \\ ${ }^{2}$ State Key Laboratory of Alternate Electrical Power System with Renewable Energy Sources \\ North China Electric Power University, Baoding,071003, China \\ a zwp_xjtu@163.com, byanshuchang1992@hotmail.com, 'c1198481006@qq.com, \\ d2316845573@qq.com, e474686958@qq.com
}

Keywords: isolated multi-element complementary microgrid, economic and environmental operation, particle swarm optimization algorithm, adaptive mutation

\begin{abstract}
Standalone multi-element complementary microgrid plays a very important role in solving the electricity problems of many areas, which are rich in renewable energy but have problems in the power supply of traditional power grid. To ensure the reliability of power supply while improving overall economic and environmental operation in micro-grid system, we have to optimize the operation of the system according to actual conditions of the system. This paper considered operating costs of the system and gas pollution emissions, established an optimization model. And the dispatch strategies are designed. Finally, utilized adaptive mutation particle swarm optimization algorithm to solve operational problems. Specific case study results verified the reasonableness and effectiveness of the algorithm.
\end{abstract}

\section{Introduction}

With the emergence of the energy crisis and environmental issues, distributed generation, especially wind and solar photovoltaic, has become a hot research topic worldwide. Microgrid is defined as an autonomous system composed of the distributed power, energy conversion devices, load monitoring and protection devices which can achieve self-control and management [1]. Microgrid can integrate a variety of distributed generation technologies effectively, to meet user requirements for higher power quality and supply reliability, giving full play to economical and environmental benefits that the renewable energy bring [2], [3]. Currently, the research on microgrid optimization operation is still in its infancy. Reference [4] studied the optimization scheduling strategy of different scenarios in the microgrid composed of photovoltaic cells and energy storage systems, improved genetic algorithm is used to verified the effectiveness of the proposed method. Reference [5] uses the improved genetic algorithm to solve economical dispatch in isolated microgrid. Reference [6] studied the best operation strategy of microgrid and uses bacterial foraging algorithm to solve microgrid optimization operation problem. Reference [7] studies microgrid optimal scheduling strategy from the economic, technology and enviromental aspects, a comprehensive scheduling strategy based on the combination of genetic algorithm and analytic hierarchy process is proposed in the condition that there are conflicts among different goals. This paper studies isolated microgrid, containing photovoltaic cells(PV), wind turbine(WT), microturbines (MTG), the diesel generator(DIE) and fuel cell(FC). Due to the fact that the reaction speed of MTG and FC is slow and that of loads may be faster, which will pose threat to normal microgrid operation and even lead to the collapse of the microgrid. Therefore, storage battery is added in this paper to ensure fast power balance. In addition, the operation cost of the system and exhaust gas pollution are considered, the operation cost is composed of fuel costs, system operation and maintenance costs and battery depreciation costs, while the energy penalty fee, the cost of power loss and power subsidies are introduced. Finally, microgrid optimization operation problem 
is solved by modified self-adaptive particle swarm algorithm

\section{Independent multivariable microgrid optimization model}

\section{A. The establishment of objective function}

Based on the local requirements and actual situation, this paper set the corresponding weights to the two objectives above and the multi-objective problem is transformed into a single goal to be addressed by linear weighting.

\section{1) Objective 1:Minimal operating costs.}

a) fuel cost

The fuel costs of MTG relates to its work efficiency[8].

$$
F_{M T G}=C \frac{1}{V_{L H V}} \frac{P_{M T G}}{\eta_{M T G}}
$$

Where the $F_{\text {MTG }}$ represents fuel cost per unit time, $C$ represents gas price, which is yuan $/ \mathrm{m} 3$, $V_{\mathrm{LHV}}$ represents low-hot value of natural gas, which is $9.7 \mathrm{kWh} / \mathrm{m} 3, \eta_{\text {MTG }}$ represents the working efficiency of MTG.

The fuel cost of FC is similar to that of MTG,

$$
F_{F C}=C \frac{1}{V_{L H V}} \frac{P_{F C}}{\eta_{F C}}
$$

Where $F_{\mathrm{FC}}$ represents fuel cost per unit time, $\eta_{F C}$ represents the working efficiency, $P_{\mathrm{FC}}$ represents output power.

The fuel cost of DIE is consumption characteristic function and it can expressed,

$$
F_{D I E}=a+b P_{D I E}+c P_{D I E}^{2}
$$

Where $\mathrm{a}, \mathrm{b}$ and $\mathrm{c}$ are fuel cost coeffients.

b) operation and maintenance costs

The microgrid operation and maintenance costs of each unit relate to each power generation unit,

$$
E=\sum_{i=1}^{N} k_{i} P_{i}
$$

Where $N$ represents the number of microsources, $P_{\mathrm{i}}$ represents the $i$ th microsource output power; $k_{\mathrm{i}}$ represents the $i$ th microsource operation and maintanence cost.

c) the depreciation costs of battery

Because the amount of charging and discharging remain constant in the life circle [9], depreciation costs are expressed as follows,

$$
C_{\text {old }}=\frac{C_{\text {rep }}}{Q_{\text {life }}}
$$

Where, $C_{\text {old }}$ is depreciation cost when the battery charge or discharge $1 \mathrm{~kW} \bullet \mathrm{h} ; C_{\text {rep }}$ is the battery replacement costs; $Q_{\text {life }}$ is the total amount of charging or discharging in the life cycle of the battery.

d) the penalty fee of energy wasting

When the battery is fully charged, if surplus electricity still occurs, the extra power has to be wasted. To avoid the waste of energy, so the penalty fee of energy wasting is introduced.

$$
C_{\text {waste }}=\int_{0}^{T} k_{\text {waste }} P_{\text {waste }}(t) d t
$$

Where $k_{\text {waste }}$ is the cofficient of energy wasting penalty (yuan/kWh), $P_{\text {waste }}(\mathrm{t})$ is the surplus power(kW).

e) the cost of power failure

For independent micro-sources, when the internal power sources and energy storage systems can not meet the loads, part of the non-essential load will be cut off, so the cost of power failure is introduced.

$$
C_{\text {loss }}=\int_{0}^{T} k_{\text {loss }} P_{\text {loss }}(t) d t
$$


Where the $k_{\text {loss }}$ represents the coefficient of power failure cost and $P_{\text {loss }}(t)$ represents the power vacancy.

f) generation subsidy

Due to the fact that the renewable generation such as wind power and photovoltaic cell is encouraged by governments and the generation subsidy is introduced [10],

$$
C_{\text {subsidy }}=k_{\text {subsidy }} \int_{0}^{T} P_{\text {subsidy }}(t) d t
$$

Where the $k_{\text {subsidy }}$ represents the subsidy coefficient (yuan $/ \mathrm{kW} \cdot \mathrm{h}$ ), $P_{\text {subsidy }}(t)$ is the output power of renewable energy $(\mathrm{kW})$.

Based on the six costs above, the overall operating cost of the system is as follows,

$$
C_{\text {total }}=F_{M T G}+F_{F C}+F_{D I E}+E+C_{\text {old }}+C_{\text {waste }}-C_{\text {subsidy }}
$$

The first objective function is

$$
f_{1}=\min C_{\text {total }}=f\left(P_{1}, P_{2}, \ldots, P_{N}\right)
$$

2) Objective 2:Minimal operating costs.

The photovoltaic cells, wind turbine, battery does not produce polluted gases, MTG, FC, DIE are the major sources of polluted gases, mainly emitting $\mathrm{CO} 2$, NOx, SOx, their ability of emitting polluted gases is measured by emission factors. In this paper, The damage caused by the various polluting gases to the environment is characterized by conversion cost factor. Thus, the pollution degree of the environment is characterized by,

$$
f_{1}=\min C_{\text {total }}=f\left(P_{1}, P_{2}, \ldots, P_{N}\right)
$$

When $i$ equals to 1,2 or $3, P_{\mathrm{i}}(\mathrm{t})$ corresponds to the output power of MTG、FC and DIE respectively. When $j$ equals to 1,2 and $3, K_{\mathrm{i}}(\mathrm{g} / \mathrm{kW} \cdot \mathrm{h})$ corresponds to the coefficients of $\mathrm{CO}_{2}, \mathrm{NO}_{\mathrm{x}}$ and $\mathrm{SO}_{\mathrm{x}}$ respectively and $S_{\mathrm{j}}$ (yuan/kg) corresponds to the coefficients of $\mathrm{CO}_{2}, \mathrm{NO}_{\mathrm{x}}$ and $\mathrm{SO}_{\mathrm{x}}$ respectively.

The second objective function is:

$$
f_{2}=\min E_{\text {harm }}=f\left(P_{1}, P_{2}, \ldots, P_{N}\right)
$$

According to local requirements and the actual situation, economical and environmental objectives are given corresponding weights, and the third objective function can be obtained by the linear weight:

$$
f_{3}=\min \left(\lambda_{1} C_{\text {total }}+\lambda_{2} E_{\text {harm }}\right)=f\left(P_{1}, P_{2}, \ldots, P_{N}\right)
$$

Where $\lambda_{1}+\lambda_{2}=1,0 \leq \lambda_{1} \leq 1$ and $0 \leq \lambda_{2} \leq 1$.

\section{$B$ Constraints}

$$
\begin{aligned}
& \text { 1) power balance constraint } \\
& \sum_{i=1}^{N} P_{d g i}(t)=P_{\text {load }}(t)+P_{B A T}(t)
\end{aligned}
$$

Where $\mathrm{N}$ represents the number of microsources; $P_{\text {dgi }}(\mathrm{t})$ represents $\underline{i t h}$ micrsource output power; $P_{\mathrm{BAT}}(\mathrm{t})$ represents charging or discharging rate of energy storage unit and its sign is a plus in charging and a minus in discharging; $P_{\text {load }}(\mathrm{t})$ is the total load.

2) Power limit constraints

$P_{i}^{\min } \leq P_{i} \leq P_{i}^{\max }, \forall i=1, \ldots, N$

Where $P_{\mathrm{i}}^{\max }$ and $P_{\mathrm{i}}^{\text {min }}$ are the maximum and minimum output power of the ith microsource, $N$ is the number of microsources.

3) Constraints on Batteries

$S O C_{\min } \leq S O C \leq S O C_{\max }$

Where $S O C_{\min }$ and $S O C_{\max }$ are the minimum and maximum of residual capacity for battery, generally $S O C_{\min }=0.1 \sim 0.2, S O C_{\max }=0.8 \sim 0.9$. In order to make battery firstly coming into use be able to charge or discharge, the value of $S O C(0)$ is set at $0.5 \sim 0.6$.

In order to prevent over charge and over discharge, the limit of maximum charge/discharge current and rate are taken into account, so the constraints on charging and discharging rate are as follows:

$$
P_{c \max } \leq P_{B A T}(t) \leq P_{d \max }
$$


Where the $P_{\text {cmax }}$ is the lower limit of the battery output power representing charging power, whose sign is a minus and the $P_{\text {cmax }}$ is the upper limit of the battery output power, representing discharging power, whose sign is a plus.

\section{The solutions}

The difference between the adaptive particle swarm algorithm and standard particle swarm algorithm is as follows.

A Inertia weight changes with the search process self-adaptively

In the standard particle swarm algorithm, inertia weight $\omega$ commonly remain constant or a decreasing trend, the adjustment for search capacity is limited, which cannot be well adapted to non-linear and high-dimensional complex practical problems. In this paper, $\omega$ is adjusted according to the difference of optimal fitness.

$$
\begin{aligned}
& \bar{f}=\frac{1}{6} \sum_{t=i-6}^{i-1} \text { fitness }(t) \\
& k=\frac{1}{6} \sum_{t=i-6}^{i-1}(\text { fitness }(t)-\bar{f})^{2} \\
& w=\left\{\begin{array}{l}
0.9, \quad i<7 \\
0.6+\text { rand } / 2, \quad i>6 \text { and } k \geq 0.01 \\
0.2+\text { rand } / 4, \quad i>6 \text { and } k<0.01
\end{array}\right.
\end{aligned}
$$

Where, fitness $(t)$ is the optimal fitness value of the t-th iteration, $\bar{f}$ is the mean value of optimal fitness value in 6 iterations; rand is uniformly distributed random number between 0 and 1 . When $k \geq 0.01$, the differences of the optimal fitness value among 6 iterations are large, representing the population is at the exploratory stage, $w$ take a larger value is in favor of speeding up the algorithm convergence. When $k<0.01$, the differences of the optimal fitness value among 6 iterations are little, $w$ take a smaller value is in favor of fine search to get accurate solution.

$B$ Use mutation in particle swarm algorithm for reference

In this paper, the advantages of genetic algorithm are used for reference, the mutation is introduced and the diversity of the population is increased to allow the algorithm to escape from local optimal solution and ultimately obtain global optimal solution.

When the population is seriously lack of diversity or optimal fitness value remain constant for a long time, then perform mutation. The average distance $D$ in each iteration characterizes the degree of population diversity. Whether the fitness value remain constant can be judged by the optimal fitness differences $k$, the formula to calculate $k$ is similar to (19).

$$
D=\frac{1}{N \cdot L} \sum_{i=1}^{N} \sqrt{\sum_{d=1}^{n}\left(x_{i d}^{t}-\overline{x_{d}}\right)^{2}}
$$

Where $N$ represents the population size, $L$ is a diagonal length of the search space, $t_{\text {xid }}$ represents the $t$ th iteration, the ith particle and dth dimensional coordinate values, $x_{\mathrm{d}}$ is the mean value of the $d$ th dimensional coordinates of all the particles.

When $D<0.001$ or $k<0.01$, mutation is performed. the process is that each particle is sorted in accordance with the quality of the fitness values [11] and take the corresponding number of particles with best fitness value to mutate.

$x_{\text {id }}^{t}=x_{\text {id }}^{t}\left[1+0.25\left(\right.\right.$ rand $_{1}-$ rand $\left.\left._{2}\right)\right]$

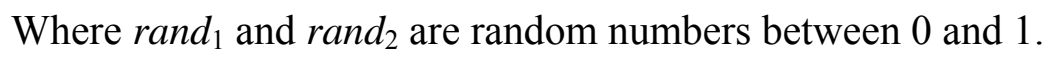




\section{Test results}

\section{A Case introduction and parameter setting}

The rated capacity of PV, WT, DIE, FC, MTG and BAT are $15 \mathrm{~kW}, 15 \mathrm{~kW}, 8 \mathrm{~kW}, 6 \mathrm{~kW}, 10 \mathrm{~kW}$ and $6 \mathrm{~kW}$ respectively in this case. The hourly data of load is shown in Fig. 1 in a day, The predicted output power of PV and WT is shown in Fig.2, the coefficients of operation and maintenance cost, the polluted gases emission and conversion cost is shown in in Table I, Table II and Table III respectively, the operation and scheduling period is $1 \mathrm{~h}$.

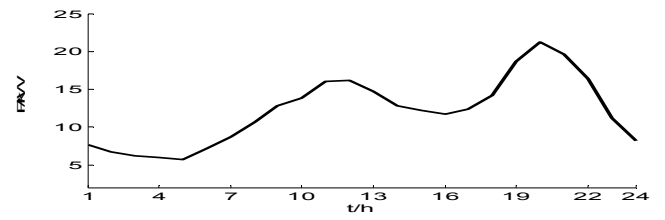

Fig.1 Forecast data of hourly load power in a day

Table 1. maintenance costs of each power source

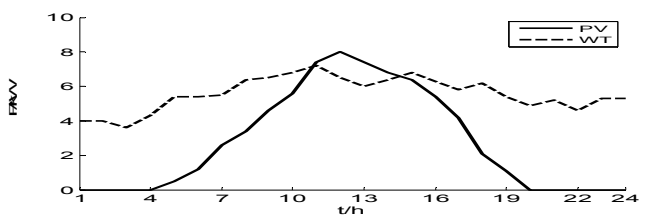

Fig.2 Forecast data of PV and WT output power in a day

Table 3. Discounted cost coefficients of polluting gases

\begin{tabular}{clll}
\hline Gas type & $\mathrm{CO}_{2}$ & $\mathrm{NO}_{\mathrm{x}}$ & $\mathrm{SO}_{\mathrm{x}}$ \\
\hline conversion cost & 0.11 & 27.54 & 6.49 \\
\hline
\end{tabular}

\begin{tabular}{ccccccc}
\hline Microsources & WT & PV & MTG & DIE & FC & BAT \\
\hline$k_{\mathrm{i}}$ & 0.0296 & 0.0096 & 0.0385 & 0.0825 & 0.04 & 0.0275
\end{tabular}

Table 2. emission coefficients of polluting gases

\begin{tabular}{ccccc}
\hline Emission & type & $\begin{array}{l}\text { MTG emission } \\
\text { Factor }(\mathrm{g} / \mathrm{kW} \cdot \mathrm{h})\end{array}$ & FC emission Factor $(\mathrm{g} / \mathrm{kW} \cdot \mathrm{h})$ & $\begin{array}{c}\mathrm{DIE} \\
\text { factor }(\mathrm{g} / \mathrm{kW} \cdot \mathrm{h})\end{array}$ \\
\hline $\mathrm{CO}_{2}$ & 1.6 & 1.625 & 1.432 \\
\hline $\mathrm{NO}_{\mathrm{x}}$ & 0.44 & 0.023 & 21.890 \\
\hline $\mathrm{SO}_{\mathrm{x}}$ & 0.008 & 0.006 & 0.406 \\
\hline
\end{tabular}

Gas is 2 yuan $/ \mathrm{m}^{3}$, the low-hot value is $9.7 \mathrm{~kW} \cdot \mathrm{h} / \mathrm{m} 3$, the generation efficiency of full cell is $50 \%$, the consumption parameters $\mathrm{a}, \mathrm{b}$ and $\mathrm{c}$ of DIE are 6, 0.012 and 0.00085 respectively, the replacement costs of battery is 90 yuan, the total amount of battery charging or discharging in the life cycle is $100 \mathrm{~kW} \cdot \mathrm{h}$, the coefficient of energy wasting penalty is 0.2 , the subsidy coefficient is 0.4 , power failure cost factor is 0.3 , the value of $S O C(0)$ is 0.5 , the range of SOC is from 0.1 to 0.8 , and the limit of battery charging or discharging is $1 \mathrm{~kW}$.

The population size is 20 , the maximum number of iterations is 200 , learning factor $\mathrm{c} 1$ and $\mathrm{c} 2$ are both 1.49445 and the mutation rate is 0.2 .

B Optimization Results and Analysis

Microgrid operates to achieve object 1, the output power each hour of DIE, FC and MTGs are shown in Fig.3, the operating cost each hour is shown in Fig.4, the total cost of a day is 118.3237 yuan, and the charge or discharge power of the battery is shown in Fig.5.

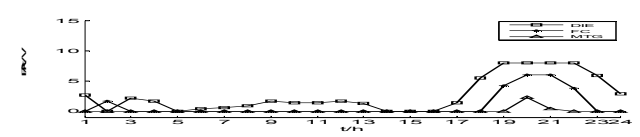

Fig.3 Hourly output of DIE, FC and MTG based on objective 1

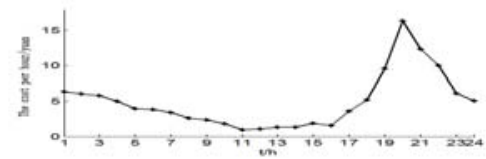

Fig.4 Hourly operating cost based on objective 1

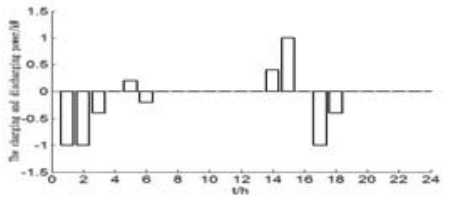

Fig. 5 Battery charge and discharge power of each hour

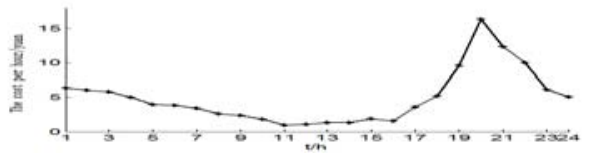

Fig.4 Hourly operating cost based based on objective 1

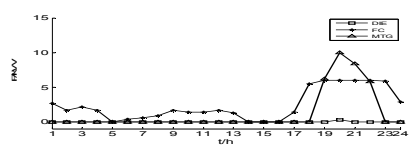

Fig.6 Hourly output of

DIE,FC and MTG based on objective 2

Microgrid operates to achieve object 2, the output power each hour of DIE,FC and MTGs are shown in Fig.6 and the charge or discharge power of the battery is shown in Fig.5.

Microgrid operates to achieve object 3, assume $\lambda_{1}=\lambda_{2}=0.5$ and then the output power of DIE, 
FC and MTG each hour are shown in Fig.7, the operating cost each hour is shown in Fig.8, the total cost of a day is 74.7315 yuan, and the charge or discharge power of the battery is shown in Fig.6.

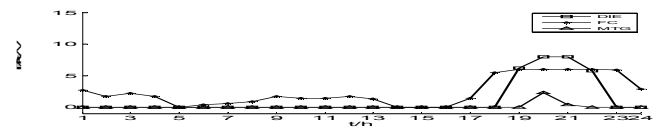

Fig.7 Hourly output of DIE、FC and MTG based on objective 3

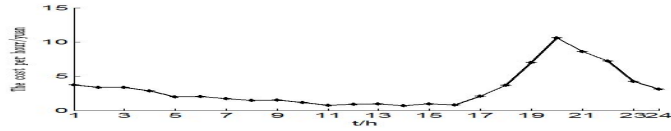

Fig. 8 Hourly cost based on objective 3

Fig. 3 to Fig. 8 shows that when the microgrid operates mainly for economical objective, PV, WT, DIE are the main power generation unit, FC and MTG are the back up power, when the system operates mainly for enviromental objective, PV, WT, FC are the main power generation units, DIE and MTG are the back-up power. the utilization of renewable energy greatly reduces the emissions of polluted gases. When power generation is greater than the load, the extra power is substantially absorbed by the battery. When power shortage occurs, battery is the first to discharge. In addition, the value of $\lambda_{1}$ and $\lambda_{2}$ can be modified to focus on different optimization objectives.

\section{Conclusion}

Multiple independent complementary microgrid play a very important role in supplying power to places where the renewable resources are abundant but it is difficult for traditional power grids to supply power. From the perspective of economic and environmental operation, considering the system's operating costs, environmental pollution, the effective optimization model is established and reasonable scheduling strategy is designed. By combining specific case, self-adaptive particle swarm algorithm with mutation is used to solve the microgrid optimization problem, which provide a theoretical basis for studying microgrid effective reasonable operation further.

\section{References}

[1] Lasseter R, Akhil A, Marnay C, Jeff Dagle, Ross Guttromson, A. Sakis Meliopoulous, Robert Yinger, Joe Eto. Integration of distributed energy resources[C]. Proceedings of Consortium for Electric Reliability Technology Solutions, Berkeley, USA, 2002.

[2] Li Peng, Zhang Ling, Wang Wei, Yang Xilei, Zhao Yishu. Application and analysis of microgrid[J]. Automation of Electric Power Systems, 2009, 33(20):109-115.

[3] Lei Jinyong, Li Zhanying, Lu Zehan, Xin Huanhai, Yang Huan. Review on the research of distributed generation technology and its impacts on electric power systems[J]. Southern Power System Technology, 2011, 5(4): 46-50.

[4] Wang Chengshan, Hong Bowen, Guo Li. Dispatch strategies of PV-battery microgrid in different scenarios[J]. Power System Technology, 2013, 37(0):1-8.

[5] Kong Shuqin, Niu Ming, Fu Xueqian, Guo Jiahuan. Research on economical operation of island microgrid[J]. Power System and Clean Energy, 2012, 28(4):57-62.

[6] R. Noroozian, H. Vahedi. Optimal management of microgrid using bacterial foraging algorithm[C]. Proceedings of ICEE, Isfahan, Iran, 2010.

[7] Zhao Yuanyuan, Ai Qian, Yu Zhiwen, He Xing. A microgrid optimal scheduling considering the assessment of various factors[J]. Power System Protection and Control, 2014, 42(23):23-30.

[8] Dennis B C, Byron Winn C. Optimal dispatch strategy in remote hybrid power systems[J]. Solar Energy, 1996,58(4-6):165-179.

[9] Ding Ming, Wang Bo, Zhao Bo, Chen Zinian. Configuration optimization of capacity of standalone PV-wind-diesel-battery hybrid microgrid[J]. Power System Technology, 2013, 37(3):575-581. 
[10]Zhang Jianhua, Su Ling, Chen Yong, Su Jing,Wang Li. Energy management of microgrid and its control strategy[J]. Power System Technology, 2011, 35(7):24-28.

[11]Mohamed F A, Koivo H N. System modeling and online optimal management of microgrid using multi-objective optimization[C]. International Conference on Clean Electrical Power, Capri, Italy: IEEE, 2007:148-153. 